\title{
Assessment of pain score and specimen adequacy for ultrasound-guided fine-needle aspiration biopsy of thyroid nodules
}

This article was published in the following Dove Press journal: Journal of Pain Research

\author{
Li-Jen Liao' \\ Wu-Chia Lo' \\ Wan-Lun Hsu² \\ Po-Wen Cheng' \\ Cheng-Ping Wang ${ }^{3}$ \\ 'Department of Otolaryngology, Far \\ Eastern Memorial Hospital, ${ }^{2}$ Genomics \\ Research Center, Academia Sinica, \\ ${ }^{3}$ Department of Otolaryngology, \\ National Taiwan University Hospital, \\ Taipei, Taiwan, Republic of China
}

Objective: The aim of this study was to evaluate pain scores and specimen adequacy for ultrasound-guided fine-needle aspiration biopsy (US-FNAB) of thyroid nodules without and with local anesthesia (LA).

Materials and methods: The US-FNAB procedure was performed on 183 patients with and without LA. One puncture was made for solid nodules, and if patients could tolerate it, a twopuncture technique was used for nodules with a cystic change. Four-point verbal rating scores were assessed by a nursing assistant after completion of US-FNAB. To be an adequate specimen, at least six groups of follicular cells are required, and each group should contain at least 10 cells. Results: Immediately after US-FNAB, $92 \%$ of patients with LA and $80 \%$ without LA reported no or mild pain $(p=0.01)$. Most patients tolerated the procedure well, with no pain $(82.5 \%)$ reported 5 minutes after the procedure. In univariate logistic regression, irregular boundary (odds ratio [OR]: $2.52,95 \%$ confidence interval [CI]: $1.04-6.06, p=0.04$ ), calcification (OR: $2.86,95 \% \mathrm{CI}$ : $1.06-7.76, p=0.04$ ), and LA (OR: $0.35,95 \%$ CI: $0.15-0.86, p=0.02$ ) were significantly associated with immediate moderate or severe pain. Specimen adequacy was significantly associated with age (OR: $0.95,95 \%$ CI: 0.92-0.97, $p<0.01$ ), heterogeneous echo-texture (OR: $1.76,95 \%$ CI: $1.23-5.17, p=0.01$ ), predominate solid architecture (OR: 2.78, 95\% CI: 1.42-5.41, $p<0.01$ ), and the use of LA (OR: $3.34,95 \% \mathrm{CI}: 1.70-6.56, p<0.01$ ). In multivariate logistic regression, patients receiving LA had lower risk of moderate or severe pain (OR: 0.25, 95\% CI: 0.09-0.67, $p=0.01$ ) and higher chances of specimen adequacy (OR: 4.84, 95\% CI: 2.17-10.7, $p<0.01$ ) compared to patients who did not receive LA.

Conclusion: US-FNAB is a safe procedure, and most patients report no pain 5 minutes after the procedure. The use of LA was associated with lower immediate pain scales and higher specimen adequacy.

Keywords: ultrasound, thyroid nodule, fine-needle aspiration, pain score, local anesthesia

\section{Background}

Thyroid nodules are very common in the general population, and the possibility of malignant transformation is relatively low. ${ }^{1}$ Before adequate management can be implemented, thyroid nodules are frequently evaluated by fine-needle aspiration biopsy (FNAB) to establish a tentative diagnosis. An ideal biopsy procedure not only affects the cytology specimen adequacy but also decreases sensations of discomfort. FNAB can be performed under palpation guidance, or it can be ultrasound guided (US-FNAB). FNAB cytology can determine the possibility of a malignancy, and the features of cytology also have prognostic value in thyroid papillary carcinoma. ${ }^{2}$ US guidance enhances the diagnostic accuracy, as it helps to direct the tip to the desired
Correspondence: Cheng-Ping Wang Department of Otolaryngology, National Taiwan University Hospital, No. 7, Chung-Shan South Road, Taipei, Taiwan, Republic of China

Email wangcp1971@gmail.com 
site. ${ }^{3}$ However, US-FNAB also brings some complications, including pain and anxiety, hemorrhage, infection, recurrent laryngeal nerve injury, and possible needle track seeding. ${ }^{4}$

Most patients are generally able to tolerate the pain and anxiety during aspiration. ${ }^{5}$ Therefore, the routine administration of local anesthesia (LA) for US-FNAB is not usually recommended. ${ }^{6,7} \mathrm{Kim}$ et $\mathrm{al}^{8}$ even reported that pain scales in patients with one needle puncture were significantly lower when no LA was used prior to US-FNAB of thyroid nodules, compared to when LA was used prior to US-FNAB. Sometimes, however, more than one puncture is necessary. Therefore, other studies routinely use LA before US-FNAB. ${ }^{9-11}$ The question of whether to use LA before US-FNAB for thyroid nodules still remains a subject of debate. Additionally, the associations of pain scores and specimen adequacy with US characteristics and LA are still unclear.

We hypothesized that the use of LA would decrease pain and cytology inadequacy rates in US-FNAB of thyroid nodules. Thus, the aim of this study was to evaluate the differences in pain levels and cytology adequacy for US-FNAB of thyroid nodules in relation to various factors.

\section{Materials and methods}

This study protocol was approved by the institutional ethics committee of the Far Eastern Memorial Hospital ethical review board (approval No. 102171-F). Every patient signed a written informed consent prior to US-FNAB.

Patients who had thyroid nodules requiring US-guided FNAB were included as the participants in our study. The group of patients with thyroid nodules who received USFNAB without LA was previously reported. ${ }^{5}$ We recruited a second group of patients with thyroid nodules who received US-FNAB with LA for comparison. No participants dropped out during the study. The sonograms were performed by two sonologists (L-J Liao and W-C Lo, both of whom have experience performing more than 1,000 US-guided FNAB procedures) using an ATL HDI 5000 (Philips Medical Systems, Bothell, WA, USA) with a high-resolution 7.5-12 MHz real-time linear-array transducer (Philips HDI 5000).

Patients with painful neck masses, those using analgesic drugs or long-term topical steroids, and those with a history of bleeding disorders were excluded from our study. All remaining patients underwent a thorough neck examination using grayscale and power Doppler ultrasonography methods. Each thyroid nodule was first assessed with grayscale sonography for morphologic parameters in the horizontal section. The anteroposterior (AP) diameter and transverse (TR) diameter were measured. The larger of the AP or TR diameter was regarded as nodular size. The echo structure was categorized as a predominantly solid component or mixed cyst. Echogenicity with respect to the surrounding soft tissue was assessed and classified as hypoechoic, isoechoic, or hyperechoic. The nodule margin was defined as regular or irregular. Microcalcification was determined as present or absent. Internal echo-texture was divided into heterogeneous or homogeneous. Vascular patterns were then surveyed by power Doppler sonography and categorized as avascular or peripheral type or marked intranodular type, which have been described in detail in previous literature. ${ }^{12,13}$ We measured the distances between the skin and the superficial surface of the thyroid nodule (Depth 1) and between the skin and the center of the thyroid nodule (Depth 2) (Figure 1). The US-FNAB procedure was performed without LA for 74 patients from March 2011 to June 2011. After a washout period, 109 patients underwent US-FNAB with LA from December 2013 to August 2015. The results from these procedures were collected for comparison. Under US guidance toward the target thyroid nodule, $1 \mathrm{~mL}$ of $2 \%$ Xylocaine (lidocaine hydrochloride) was injected subcutaneously over the aspiration site using a $27-\mathrm{G}$ needle and a $3 \mathrm{~mL}$ syringe prior to the US-FNAB. US-FNAB was performed using the free-hand technique by positioning the array probe parallel to the needle for guidance and inserting a 22-G needle into the thyroid nodules. Gentle compression with a cotton ball was performed for 15 minutes after aspiration.

For solid nodules, a single aspiration was performed, while two procedures were performed for nodules with a partial cystic change. Fluid in the cystic part was first sucked

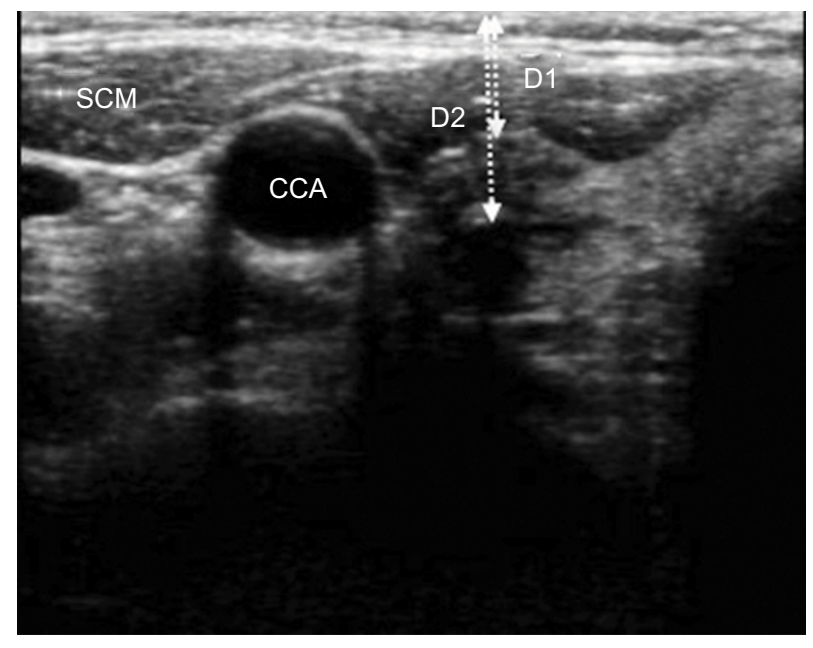

Figure I Depth I (DI) measures the distance from the skin to the surface of the thyroid nodule, and depth 2 (D2) represents the distance from the skin to the center of the nodule.

Abbreviations: SCM, sternocleidomastoid muscle; CCA, common carotid artery. 
out, and a second aspiration was performed for the solid part at a later time. One aspiration from six passes was used to acquire adequate material for the cytology assessment. The size of the thyroid nodules and total puncture time were recorded. The procedure time was recorded starting from the insertion of the first needle until the withdrawal of the last needle for cytology.

Four-point verbal rating scores were assessed by a nursing assistant immediately after and 5 minutes after the completion of US-FNAB. A score of 0 represented no pain; 1 , mild pain; 2, moderate pain; and 3, severe pain. Patients were asked for their retrospective judgments about whether they felt that they needed LA for the biopsy.

For specimen adequacy, the same pathologist checked the cytology. For a specimen to be adequate, at least six groups of follicular cells were required, and each group had to be composed of at least 10 cells. A macrophage-only sample was regarded as unsatisfactory according to the Bethesda system for reporting thyroid cytopathology. ${ }^{14}$

\section{Statistical analysis}

Continuous data were expressed as the mean \pm standard deviation. Categorical data were expressed as the number and percentage. The immediate and 5 minutes pain scores were classified into no/mild and moderate/severe groups and were assessed with Pearson's $\chi^{2}$ tests. Procedure times and puncture numbers were compared using the unpaired Student's $t$-test. Logistic regression analysis was used to analyze various factors related to pain scores and specimen adequacy. Factors that were related to pain scores and specimen adequacy in univariate analyses were further included in multivariate analyses to find independent factors. A twotailed value of $p<0.05$ was considered to indicate statistical significance. Statistical analyses were performed using STATA 12.0 (StataCorp LP, College Station, TX, USA).

\section{Results}

A total of 183 patients were enrolled (mean age: 52.0 \pm 12.6 years; range: $23-83$ years), including 138 women and 45 men. Seventy-four US-FNAB procedures were performed without LA, and 109 US-FNAB procedures were performed with LA. The demographics and US characteristics of patients with and without LA are summarized in Table 1. There were no differences in gender, age, and depth of the nodules $(p>0.05)$. However, there were significant differences in heterogeneous internal echo, calcification, and intranodular vascular pattern between patients with and without LA $(p<0.05)$.

For pain score analyses, most patients tolerated the procedure well and suffered no pain $(82.5 \%, 151 / 183)$ at 5 minutes after the puncture. Only 3 of the 183 patients (1.6\%) reported experiencing moderate pain 5 minutes after the aspiration, despite LA (Table 2). Patients who received LA had significantly lower immediate pain scores; $20 \%$ of patients without LA reported moderate to severe pain, while $8 \%$ of patients with LA reported moderate to severe pain $(p=0.01)$. None of the patients without LA versus $3 \%$ of patients with LA reported moderate to severe pain 5 minutes after aspiration, although the difference was not statistically significant $(p=0.15)$. The use of LA was also associated with higher rates of cytology adequacy $(60 \%$ versus $83 \%, p<0.01)$.

Logistic regression was used to assess multiple factors related to immediate pain scores (Table 3). For the univariate analysis, irregular boundary (odds ratio [OR]: 2.52, 95\% confidence interval $[\mathrm{CI}]: 1.04-6.06, p=0.04)$, calcification (OR: 2.86, 95\% CI: 1.06-7.76, $p=0.04$ ), and LA (OR: 0.35, $95 \%$ CI: $0.15-0.86, p=0.02)$ were significantly associated

Table I Characteristics of patients with thyroid nodules who received US-FNAB

\begin{tabular}{lll}
\hline Characteristics & Without LA (n=74) & With LA (n=I09) \\
\hline Sex (F/M) & $55 / 19$ & $83 / 26$ \\
Age & $51.5 \pm 13.9$ & $52.3 \pm 12.7$ \\
Size & $2.2 \pm 1.0$ & $2.0 \pm I .1$ \\
Depth & & 0.78 \\
Depth I (cm) & $0.6 \pm 0.3$ & $0.7 \pm 0.3$ \\
Depth 2 (cm) & $1.4 \pm 0.4$ & $1.4 \pm 0.5$ \\
US characteristics & & 0.13 \\
Architecture (cyst/solid) & $36 / 38$ & 0.21 \\
Boundary (regular/irregular) & $55 / 19$ & $77 / 32$ \\
Internal echo (homo/heter) & $36 / 38$ & $71 / 38$ \\
Echogenicity (hypo/iso) & $57 / 17$ & $92 / 17$ \\
Calcification (absent/present) & $69 / 5$ & $87 / 22$ \\
Intranodular vascular pattern (absent/present) & $61 / 13$ & $108 / 1$ \\
\hline
\end{tabular}

Note: Date are presented as mean \pm standard deviation unless otherwise specified.

Abbreviations: LA, local anesthesia; US-FNAB, ultrasound-guided fine-needle aspiration biopsy; $F$, female; $M$, male. 
Table 2 Comparisons of pain scores, cytology adequacy, procedure times, and aspiration numbers between patients with and without LA

\begin{tabular}{|c|c|c|c|}
\hline Characteristics & Without LA $(n=74)$ & With LA $(n=109)$ & $p$-value \\
\hline \multicolumn{4}{|l|}{ Immediately after aspiration } \\
\hline \multicolumn{4}{|l|}{ VRS } \\
\hline No or mild pain & $59(80 \%)$ & 100 (92\%) & 0.01 \\
\hline Moderate or severe pain & $15(20 \%)$ & $9(8 \%)$ & \\
\hline \multicolumn{4}{|l|}{5 minutes after aspiration } \\
\hline \multicolumn{4}{|l|}{ VRS } \\
\hline No or mild pain & $74(100 \%)$ & $106(97 \%)$ & 0.15 \\
\hline Moderate or severe pain & 0 & $3(3 \%)$ & \\
\hline Adequacy rate $(\mathrm{n} \%, 95 \% \mathrm{Cl})$ & $45 / 75$ (60\%, 49\%-7I\%) & $90 / 109$ (83\%, 75\%-90\%) & $<0.01$ \\
\hline Procedure time (seconds) & $32.9 \pm 15.0$ & $24.1 \pm 16.9$ & $<0.01$ \\
\hline Number of aspirations & $1.12 \pm 0.4$ & $1.63 \pm 0.48$ & $<0.01$ \\
\hline
\end{tabular}

Note: Date are presented as mean \pm standard deviation unless otherwise specified.

Abbreviations: $\mathrm{Cl}$, confidence interval; LA, local anesthesia; VRS, verbal rating scale.

Table 3 Logistic regression of pain scores (VRS)

\begin{tabular}{|c|c|c|c|c|c|c|}
\hline \multirow[t]{2}{*}{ Variables } & \multicolumn{3}{|c|}{ Univariate } & \multicolumn{3}{|c|}{ Multivariate } \\
\hline & OR & $95 \% \mathrm{Cl}$ & p-value & OR & $95 \% \mathrm{Cl}$ & $p$-value \\
\hline $\operatorname{Sex}(M / F)$ & 0.78 & $0.27-2.23$ & 0.65 & 0.97 & $0.31-3.03$ & 0.96 \\
\hline Age (years) & 1.00 & $0.97-1.04$ & 0.83 & 1.00 & $0.97-1.04$ & 0.93 \\
\hline Size $(\mathrm{cm})$ & 0.80 & $0.5 \mathrm{I}-\mathrm{I} .24$ & 0.32 & & & \\
\hline Irregular boundary & 2.52 & $1.04-6.06$ & 0.04 & 2.09 & $0.80-5.49$ & 0.13 \\
\hline Heterogeneous internal echo & 2.19 & $0.92-5.24$ & 0.08 & & & \\
\hline Hypo echogenicity & 0.86 & $0.27-2.70$ & 0.80 & & & \\
\hline Calcification & 2.86 & $1.06-7.76$ & 0.04 & 3.56 & $1.08-11.68$ & 0.04 \\
\hline Predominately solid architecture & 1.53 & $0.62-3.79$ & 0.35 & & & \\
\hline Intranodular vascular mark pattern & I.II & $0.23-5.31$ & 0.89 & & & \\
\hline Procedure time (seconds) & 1.01 & $0.98-1.03$ & 0.65 & & & \\
\hline Depth I (cm) & 1.00 & $0.25-3.93$ & 1.00 & & & \\
\hline Depth $2(\mathrm{~cm})$ & 0.84 & $0.3 \mathrm{I}-2.24$ & 0.72 & & & \\
\hline LA (with/without) & 0.35 & $0.15-0.86$ & 0.02 & 0.25 & $0.09-0.67$ & 0.01 \\
\hline
\end{tabular}

Abbreviations: $\mathrm{Cl}$, confidence interval; LA, local anesthesia; OR, odds ratio; VRS, verbal rating scale; F, female; $M$, male.

with immediate moderate or severe pain. After adjusting for multiple factors, the use of anesthesia was still an independent protective factor against moderate or severe pain (OR: 0.25 , 95\% CI: $0.09-0.67, p=0.01$ ) (Table 3). Pain score was not related to the depth of the thyroid nodules.

We further analyzed specimen adequacy using univariate and multivariate logistic regression. The results from these analyses are shown in Table 4. In univariate analysis, the adequacy rates were associated with age (OR: 0.95, 95\% CI: 0.92-0.97, $p<0.01$ ), heterogeneous echo-texture (OR: 1.76, 95\% CI: 1.23-5.17, $p=0.01$ ), predominate solid architecture (OR: 2.78 , 95\% CI: $1.42-5.41, p<0.01$ ), and the use of LA (OR: 3.34 , 95\% CI: $1.70-6.56, p<0.01)$. Under multivariate analysis, age (OR: 0.94, 95\% CI: 0.90-0.97, $p<0.01$ ), heterogeneous echotexture (OR: $2.91,95 \% \mathrm{CI}: 1.23-6.87, p=0.02)$, predominate solid architecture (OR: 2.73, 95\% CI: 1.26-5.94, $p=0.01$ ), and the use of LA (OR: 4.84, 95\% CI: 2.17-10.7, $p<0.01$ ) were all independent factors related to specimen adequacy.

\section{Discussion}

Thyroid US-FNAB is a simple and reliable procedure in the management of thyroid nodules. Complications related to the procedure have been reported in some patients, including pain, hemorrhage, infection, recurrent laryngeal nerve injury, and needle track seeding. ${ }^{4}$ Pain sensations for US-FNAB are related to the depth of the nodule, ${ }^{15}$ number of nodules biopsied, ${ }^{16}$ and use of LA, ${ }^{7,17}$ but are not related to large needle size. ${ }^{18}$ Some reports have suggested that US-FNAB should be performed without LA when one needle puncture is used. However, multiple punctures are sometimes necessary, especially for nodules with a cystic change.

Some studies have assessed factors related to specimen adequacy. Cystic change, old age, small size, hypoechogenicity with a hard pattern, and operator experience were reported as factors contributing to nondiagnosis. ${ }^{19}$ However, no studies have explored the correlation between nodule depth or use of anesthesia and specimen adequacy. 
Table 4 Logistic regression of specimen adequacy

\begin{tabular}{|c|c|c|c|c|c|c|}
\hline \multirow[t]{2}{*}{ Variables } & \multicolumn{3}{|c|}{ Univariate } & \multicolumn{3}{|c|}{ Multivariate } \\
\hline & OR & $95 \% \mathrm{Cl}$ & $p$-value & OR & $95 \% \mathrm{Cl}$ & $p$-value \\
\hline $\operatorname{Sex}(M / F)$ & 0.5 & $0.25-1.05$ & 0.07 & 0.72 & $0.31-1.67$ & 0.44 \\
\hline Age (years) & 0.95 & $0.92-0.97$ & $<0.01$ & 0.94 & $0.90-0.97$ & $<0.01$ \\
\hline Size $(\mathrm{cm})$ & 1.04 & $0.76-1.43$ & 0.81 & & & \\
\hline Irregular boundary & 1.76 & $0.8 I-3.86$ & 0.16 & & & \\
\hline Heterogeneous internal echo & 2.52 & $1.23-5.17$ & 0.01 & 2.91 & $1.23-6.87$ & 0.02 \\
\hline Hypo echogenicity & 1.26 & $0.53-3.01$ & 0.6 & & & \\
\hline Calcification & 1.36 & $0.5 I-3.60$ & 0.53 & & & \\
\hline Predominately solid architecture & 2.78 & $|.42-5.4|$ & $<0.01$ & 2.73 & $1.26-5.94$ & 0.01 \\
\hline Intranodular vascular mark pattern & 0.93 & $0.27-3.10$ & 0.9 & & & \\
\hline Procedure time (seconds) & 0.98 & $0.96-1.00$ & 0.21 & & & \\
\hline Depth I (cm) & 0.94 & $0.33-2.63$ & 0.43 & & & \\
\hline Depth 2 (cm) & 0.75 & $0.36-1.54$ & 0.43 & & & \\
\hline LA (with/without) & 3.34 & $1.70-6.56$ & $<0.01$ & 4.84 & $2.17-10.7$ & $<0.01$ \\
\hline
\end{tabular}

Abbreviations: $\mathrm{Cl}$, confidence interval; $\mathrm{LA}$, local anesthesia; $\mathrm{OR}$, odds ratio; $\mathrm{F}$, female; $\mathrm{M}$, male.

In our study, as pain scores after 5 minutes were mostly no or mild (Table 2), we included only immediate pain scores in the analysis. We found that the use of LA was associated with significantly lower immediate pain scores. We also found that irregular boundary and calcification were positively related to the pain score, while the use of LA (OR: $0.35,95 \% \mathrm{CI}$ : $0.15-0.86, p=0.02$ ) was negatively related to moderate and severe pain. Because US characteristics were differently distributed between these two groups, further adjustment for these factors was necessary to confirm the effect of LA. (Table 1) After adjusting for multiple factors, the use of anesthesia was the only factor that was negatively related to the pain score (OR: $0.25,95 \% \mathrm{CI}$ : $0.09-0.67, p=0.01$ ).

For specimen adequacy, older age was associated with a higher nondiagnostic rate (OR: $0.95,95 \%$ CI: $0.92-0.97$, $p<0.01$ ), while heterogeneous echo-texture (OR: $1.76,95 \%$ CI: $1.23-5.17, p=0.01)$, predominate solid architecture (OR: $2.78,95 \%$ CI: $1.42-5.41, p<0.01$ ), and the use of LA (OR: $3.34,95 \%$ CI: $1.70-6.56, p<0.01$ ) was associated with a lower nondiagnostic rate. After adjusting for multiple factors, the use of anesthesia (OR: 4.84, 95\% CI: 2.17-10.7, $p<0.01$ ) was still an independent factor related to specimen adequacy. In addition, we found that predominately solid architecture was independently associated with high rates of specimen adequacy, while predominate cystic architecture was associated with lower rates of specimen adequacy (Table 4).

In one previously reported study, pain scales were significantly lower in patients without LA for one-puncture procedures. ${ }^{8}$ Because we usually performed multiple punctures for nodules with a cystic change if the patient could tolerate it, we analyzed pain scores, specimen adequacy rates, and procedure time according to puncture numbers. In our practice, one-puncture aspiration was performed for solid nodules, while two punctures were made for nodules with a cystic change, if patient could tolerate it. We found that patients with LA had more puncture numbers compared to patients without LA (mean 1.63 versus $1.1, p<0.01$ ), and multiple punctures were associated with better specimen adequacy.

From the view point of specimen adequacy, the use of LA can increase the adequacy rate in cystic nodules without producing a difference in pain scores. Therefore, we suggest the use of LA for nodules with a cystic change due to need for multiple punctures. For solid nodules, the use of LA did not increase the specimen adequacy rate but decreased the procedure time and immediate pain, though the pain scores were mild and most patients tolerated the pain well, as in our previous study. The routine use of LA for solid nodules may not be necessary for one-puncture procedures.

The strength of this study is that we found multiple factors that were associated with immediate pain scores and specimen adequacy, and decisions on whether or not to use LA can be made according to these factors. There are still some potential limitations of this study. First, this is an observational study and no randomization or blinding regarding the anesthesia administration was used. A randomized study that compares the use of LA to not using LA would offer more robust evidence. Second, we only used a parallel method procedure and not a perpendicular approach, and we also only used an aspiration technique and not a capillary technique. These techniques may also have an impact on the specimen adequacy. Third, none of our patients received definite surgery, and therefore the gold standard pathology was not available for each patient. Lastly, we did not have information on individuals who declined to participate, and 
we also did not collect detailed sociodemographic data. Thus, we could not examine distributions of sociodemographic data between participants and nonparticipants, or between participants with and without LA. It is possible that the distribution of sociodemographic factors may be different between the two groups, and this should be adjusted for in future models.

\section{Conclusion}

US-FNAB is a safe procedure, and most patients have no pain 5 minutes after the procedure. The use of LA was associated with lower immediate pain scores and higher specimen adequacy.

\section{Acknowledgments}

This work was supported by grants from the Far Eastern Memorial Hospital and the National Taiwan University Hospital Joint Research Programs (104-FTN01 \& 105-FTN06).

\section{Disclosure}

The authors report no conflicts of interest in this work.

\section{References}

1. Burguera B, Gharib H. Thyroid incidentalomas. Prevalence, diagnosis, significance, and management. Endocrinol Metab Clin North Am. 2000;29(1):187-203.

2. Shih SR, Li HY, Hsiao YL, Chang TC. Prognostic significance of cytologic features in fine-needle aspiration cytology samples of papillary thyroid carcinoma: preliminary report. Thyroid. 2006;16(8):775-780.

3. Cesur M, Corapcioglu D, Bulut S, et al. Comparison of palpation-guided fine-needle aspiration biopsy to ultrasound-guided fine-needle aspiration biopsy in the evaluation of thyroid nodules. Thyroid. 2006;16(6):555-561.

4. Polyzos SA, Anastasilakis AD. Clinical complications following thyroid fine-needle biopsy: a systematic review. Clin Endocrinol. 2009;71(2):157-165.

5. Lo WC, Cheng PW, Wang CT, Yeh ST, Liao LJ. Pain levels associated with ultrasound-guided fine-needle aspiration biopsy for neck masses. Head Neck. 2014;36(2):252-256.
6. Gharib H, Papini E, Valcavi R, et al. American Association of Clinical Endocrinologists and Associazione Medici Endocrinologi medical guidelines for clinical practice for the diagnosis and management of thyroid nodules. Endocr Pract. 2006;12(1):63-102.

7. Gursoy A, Ertugrul DT, Sahin M, Tutuncu NB, Demirer AN, Demirag NG. The analgesic efficacy of lidocaine/prilocaine (EMLA) cream during fine-needle aspiration biopsy of thyroid nodules. Clin Endocrinol. 2007;66(5):691-694.

8. Kim DW, Rho MH, Kim KN. Ultrasound-guided fine-needle aspiration biopsy of thyroid nodules: is it necessary to use local anesthesia for the application of one needle puncture? Korean J Radiol. 2009;10(5):441-446.

9. Robitschek J, Straub M, Wirtz E, Klem C, Sniezek J. Diagnostic efficacy of surgeon-performed ultrasound-guided fine needle aspiration: a randomized controlled trial. Otolaryngol Head Neck Surg. 2010;142(3):306-309.

10. Smith RB. Ultrasound-guided procedures for the office. Otolaryngol Clin North Am. 2010;43(6):1241-1254, vi.

11. Kim MJ, Kim EK, Park SI, et al. US-guided fine-needle aspiration of thyroid nodules: indications, techniques, results. Radiographics. 2008;28(7):1869-1886; discussion 1887.

12. Cappelli C, Castellano M, Pirola I, et al. The predictive value of ultrasound findings in the management of thyroid nodules. QJM. 2007;100(1):29-35.

13. Rago T, Vitti P, Chiovato L, et al. Role of conventional ultrasonography and color flow-doppler sonography in predicting malignancy in 'cold' thyroid nodules. Eur J Endocrinol. 1998;138(1):41-46.

14. Cibas ES, Ali SZ. The Bethesda system for reporting thyroid cytopathology. Am J Clin Pathol. 2009;132(5):658-665.

15. Toman H, Ozkul F, Erbag G, et al. Effects of fine-needle aspiration biopsy (FNAB) nodule depth on pain score. Ir J Med Sci. 2016;185(3): 673-676.

16. Leboulleux S, Borget I, Labro S, etal. Frequency and intensity of pain related to thyroid nodule fine-needle aspiration cytology. Thyroid. 2013;23(9): $1113-1118$

17. Gursoy A, Ertugrul DT, Sahin M, Tutuncu NB, Demirer AN, Demirag NG. Needle-free delivery of lidocaine for reducing the pain associated with the fine-needle aspiration biopsy of thyroid nodules: time-saving and efficacious procedure. Thyroid. 2007;17(4):317-321.

18. Carpi A, Rossi G, Nicolini A, Iervasi G, Russo M, Mechanick J. Does large needle aspiration biopsy add pain to the thyroid nodule evaluation? PLoS One. 2013;8(3):e58016.

19. Lee YH, Baek JH, Jung SL, et al. Ultrasound-guided fine needle aspiration of thyroid nodules: a consensus statement by the Korean Society of Thyroid Radiology. Korean J Radiol. 2015;16(2):391-401.
Journal of Pain Research

\section{Publish your work in this journal}

The Journal of Pain Research is an international, peer reviewed, open access, online journal that welcomes laboratory and clinical findings in the fields of pain research and the prevention and management of pain. Original research, reviews, symposium reports, hypothesis formation and commentaries are all considered for publication.

\section{Dovepress}

The manuscript management system is completely online and includes a very quick and fair peer-review system, which is all easy to use. Visit http://www.dovepress.com/testimonials.php to read real quotes from published authors. 